\title{
A study of the suppressive effect on human pancreatic adenocarcinoma cell proliferation and angiogenesis by stable plasmid-based siRNA silencing of c-Src gene expression
}

\author{
$\mathrm{XIN} \mathrm{ZHAO}^{1 *}$, DE-CHUN LI ${ }^{1 *}, \mathrm{HUA} \mathrm{ZHAO}^{1 *}, \mathrm{ZHI} \mathrm{LI}^{2 *}, \mathrm{JIAN}-X I N \mathrm{WANG}^{3}$, \\ DONG-MING ZHU ${ }^{1}$, JIN ZHOU ${ }^{1}$ and JIAN-NONG CEN ${ }^{4}$
}

Departments of ${ }^{1}$ General Surgery and ${ }^{2}$ Intervention Radiology, The First Affiliated Hospital of Soochow University, Suzhou;

${ }^{3}$ Department of General Surgery, The Third Affiliated Hospital of Nantong University, Nantong;

${ }^{4}$ Jiangsu Institute of Hematology, Soochow University, Suzhou, P.R. China

Received September 27, 2011; Accepted December 6, 2011

DOI: 10.3892/or.2011.1602

\begin{abstract}
The non-receptor protein tyrosine kinase c-Src regulates diverse biological processes by associating with multiple signaling and structural molecules. Overexpression of c-Src occurs in pancreatic cancer and can be predictive of poor prognosis. The aim of this study was to investigate the inhibitory effects of plasmid-based siRNAs targeting the human c-Src gene on proliferation and angiogenesis in the human pancreatic adenocarcinoma cell line Panc-1. Three siRNAs targeting the c-Src gene were transfected into the Panc-1 pancreatic adenocarcinoma cell line mediated by Lipofectamine. Transfection efficiency was assessed by flow cytometry. Real-time quantitative PCR (RQ-PCR) was employed to detect the expression of c-Src mRNA, and the most effective siRNA was chosen to be cloned into a plasmid. Two single-strand DNA templates were designed according to the most effective siRNA sequences. The short hairpin RNA (shRNA) plasmid targeting c-Src with pGPU6/green fluorescent protein (GFP)/Neo vector psiRNAc-Src was constructed. Sequencing was performed to check whether the plasmid was constructed correctly. Panc-1 cells were transfected with psiRNA-c-Src and the negative control plasmid (psiRNA-N), respectively. Following selection with G418, the transfected monoclonal cells were chosen. GFP was evaluated by flow cytometry and fluorescence microscopy to estimate transfection efficiency. RQ-PCR and western blotting were used to detect c-Src silencing efficiency. To verify the effects of gemcitabine chemoresistance of c-Src expression, MTT assay was performed. ELISA was used to determine VEGF levels in culture supernatants. In a nude mouse model,
\end{abstract}

Correspondence to: Dr De-Chun Li, Department of General Surgery, The First Affiliated Hospital of Soochow University, 188 Shizi Street, Suzhou 215006, P.R. China

E-mail: dechunli.soochowedu@yahoo.cn

${ }^{*}$ Contributed equally

Key words: c-Src, pancreatic cancer, proliferation, angiogenesis, siRNA tumor growth was studied, c-Src, VEGF expression and microvessel density in tumor tissue were measured by immunohistochemistry. The transfection efficiency of siRNA in the Panc-1 cell line was above $90 \%$, the most effective siRNA could suppress expression of the c-Src gene with an inhibition efficiency of $86.1 \%$. Sequencing confirmed that the c-Src siRNA plasmid was successfully constructed. MTT assay indicated that the effect of gemcitabine-induced cytotoxicity was markedly increased in the psiRNA-c-Src group $(\mathrm{P}<0.05)$. Meanwhile, the expression of VEGF in vitro was reduced significantly $(\mathrm{P}<0.05)$ in the psiRNA-c-Src group. In nude mice bearing tumors, $\mathrm{c}-\mathrm{Src}$, VEGF expression and MVD were decreased in tumors produced from psiRNA-c-Src transfected cells $(\mathrm{P}<0.05)$. In summary, the siRNA expression constructs targeting c-Src could specifically suppress c-Src expression, inhibit VEGF expression, inhibit cell proliferation and enhance gemcitabine chemosensitivity in vitro. C-Src gene silencingwas able to inhibit angiogenesis of tumors in vivo. These findings demonstrate that the c-Src targeting gene silencing approach has the potential to serve as a novel tool for pancreatic carcinoma treatment.

\section{Introduction}

Pancreatic carcinoma remains a challenge to clinicians. The anatomical complexity and late diagnosis have led to a disappointingly low resectability rate of around $10-20 \%$, especially in pancreas disease center. Moreover, even if it is possible to resect the tumor with clear margin, early recurrence and metastasis are frequently observed. The overall 5 year survival rates are reported as below $5 \%$ (1). No adjuvant treatments have shown success in improving survival until now. Clinically chemoresistance is one of the major causes for chemotherapeutic treatment failure in pancreatic adenocarcinoma patients. Standard chemotherapeutic agents only have marginal effect on patient survival. Angiogenesis is necessary for successful tumor growth $(2,3)$, and inhibition of VEGF represents the most validated antiangiogenic approach described thus far $(4,5)$. Because of the high mortality associated with pancreatic adenocarcinoma, it is essential that therapeutic regimens be 
developed to inhibit tumor growth, increase chemosensitivity of chemotherapeutics, and restrain angiogenesis of tumors. So new approaches including gene therapy are required to improve treatment results $(6,7)$.

The progression of pancreatic adenocarcinoma has been associated with deregulation of several signaling molecules. One of the potential therapeutic targets receiving considerable recent attention is activation of $\mathrm{c}-\mathrm{Src}$, a non-receptor protein tyrosine kinase. $\mathrm{c}-\mathrm{Src}$ regulate diverse biological processes by associating with multiple signaling and structural molecules. Overexpression of c-Src occurs in many solid tumors, often at later stages of disease, and can be predictive of poor prognosis. RNA interference (RNAi) has emerged as a powerful tool to induce loss-of-function phenotypes by post-transcriptional silencing of gene expression $(8,9)$. At present, it was successfully used in the research of gene function and the correlation of upper and downstream molecule from signal transmission system, and it might provide a new strategy for tumor therapy. Plasmid vectors have provided a huge advancement in technology and seem to offer the means to achieve relatively high levels of gene transfer in vitro and in vivo. In this study, we used the plasmid vector system to deliver a specially designed small hairpin RNA for human c-Src gene into pancreatic carcinoma cell line Panc-1 to observe the gene therapy effects on proliferation and angiogenesis.

\section{Materials and methods}

siRNA, cells, reagents and mice. We designed three doublestrand siRNA oligonucleotide targeting c-Src gene by the software on line WMG and Qiagen, based on the published sequence of c-Src (GenBank, BC011566). One pair of negative control siRNA ( sequences were without obvious homology to human gene sequences (Tables I and II). At the 3'-end, it was labeled with fluorescein isothiocyanate (FITC) for transfection rate evaluation. All the siRNA sequences were finally blasted to avoid silencing other unrelated genes. They were all synthesized by Shanghai GenePharma Co., Ltd. (China).

The Panc-1 cell line was obtained from Shanghai cell repository of Chinese Acadamy of Science (China). The cells were cultured in Dulbecco's modified Eagle's medium (DMEM; Sigma, St. Louis, MO) supplemented with $10 \%$ fetal bovine serum (FBS), at $37^{\circ} \mathrm{C}$ in a humidified atmosphere containing $5 \% \mathrm{CO}_{2}$. The cultures were passaged 2 or 3 times weekly to maintain log-phase growth.

TRIzol reagent and MMLV were purchased from Gibco-BRL (Carlsbad, CA). TaqDNA polymerase, dNTPs, and DNA marker were obtained from Takara (Shanghai, China). Monoclonal mouse anti-human c-Src, VEGF and CD34 were purchased from Santa Cruz Biotechnology, Inc. (Santa Cruz, CA). 3-(4,5-dimethylthiazol-2-thiazolyl)-2,5 -diphenyltetrazolium bromide (MTT) was purchased from Sigma. BALB/c nude mice were obtained from Shanghai Experimental Animal Center (Shanghai, China) and maintained according to the Animal Research Committee guidelines at Soochow University (Suzhou, China).

siRNA transfection. Twenty-four hours before transfection, cells were diluted in fresh media without antibiotics and
Table I. Target sequence of c-Src gene.

\begin{tabular}{lc}
\hline siRNA & siRNA target sequence \\
\hline siRNA-1 & 5'-AACAAGAGCAAGCCCAAGGAT-3' \\
siRNA-2 & 5'-AAAGTGAGACCACGAAAGGTG-3' \\
siRNA-3 & 5'-AAGGGGAGTTTGCTGGACTTT-3'
\end{tabular}

Table II. Synthetic c-Src siRNA sequence.

\begin{tabular}{lc}
\hline siRNA & \multicolumn{1}{c}{ Synthetic siRNA sequence } \\
\hline siRNA-1 & F: 5'-CAAGAGCAAGCCCAAGGAUtt-3' \\
& R: 5'-AUCCUUGGGCUUGCUCUUGtt-3' \\
siRNA-2 & F: 5'-AGUGAGACCACGAAAGGUGtt-3' \\
& R: 5'-AGUGAGACCACGAAAGGUGtt-3' \\
siRNA-3 & F: 5'-GGGGAGUUUGCUGGACUUUtt-3' \\
& R: 5'-AAAGUCCAGCAAACUCCCCtt-3' \\
siRNA-N & F: 5'-UUCUCCGAACGUGUCACGUtt-3' \\
& R: 5'-ACGUGACACGUUCGGAGAAtt-3' \\
\hline
\end{tabular}

F, forward; R, reverse.

Table III. Sequences of primer for RT-PCR.

\begin{tabular}{lc}
\hline Genes & Sequences \\
\hline c-Src & F: 5'-GCCTACTACTCCAAACACGC-3' \\
& R: 5'-GTAAATGGGCTCCTCTGAAA-3' \\
$\beta$-actin & F: 5'-AGCGAGCATCCCCCAAAGTT-3' \\
& R: 5'-GGGCACGAGGGCTCATCATT-3'
\end{tabular}

F, forward; R, reverse.

transferred to 6-well plates. Panc-1 cells grown to a confluence of 50-60\% were transfected with 50-200 nmol/1 (final concentration) of siRNA per well using Lipofectamine 2000 and Opti-MEM (Invitrogen, Karlsruhe, Germany) media according to the manufacturer's recommendations. After transfection for $6 \mathrm{~h}$, flow cytometry was used to detect the transfection efficiency and fluorescence microscope was used to observe the cells which released fluorescence.

Real-time quantitative RT-PCR. Total cytoplasmic RNA was isolated after transfection using TRIzol reagent (Gibco-BRL, Life Technologies, USA). Then $2 \mu \mathrm{g}$ of total-RNA was converted to cDNA in $40 \mu \mathrm{l}$ and stored at $-20^{\circ} \mathrm{C}$ until use. The transcriptional level of c-Src was analysed using the MJ Research Opticon $^{\mathrm{TM}} 2$ DNA engine system with SYBR-Green fluorochrome. $\beta$-actin served as an internal control. The sequences of primers are shown in Table III. PCR was carried out with cDNA derived from 50 ng of RNA, 1 unit Taq polymerase and reaction kits in a final volume of $25 \mu 1$. Each cycle 


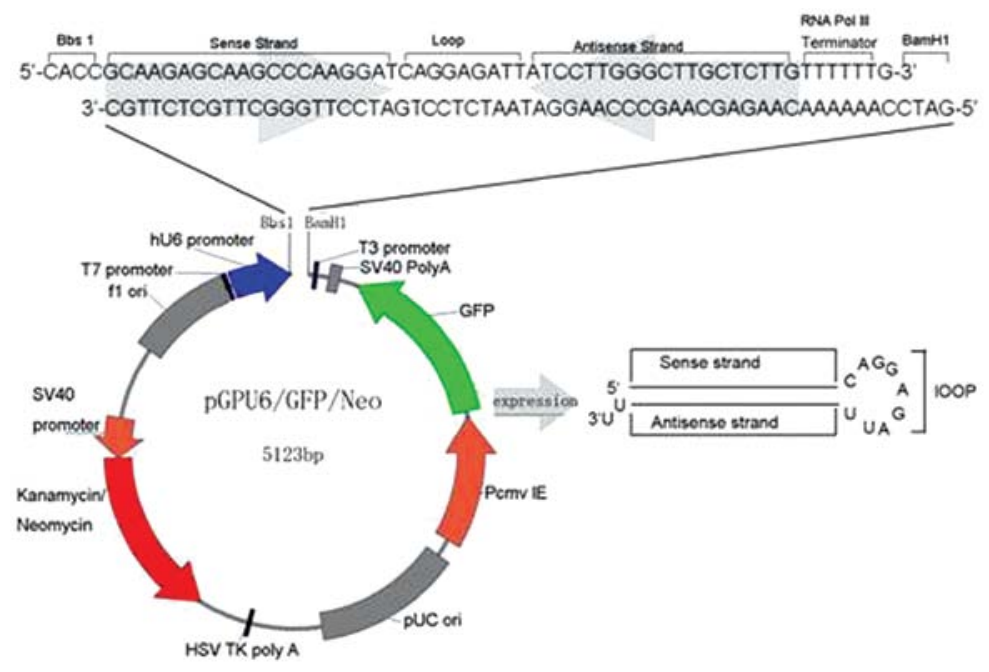

Figure 1. Structure of the recombinant vector: psiRNA-c-Src.

of PCR include $15 \mathrm{sec}$ of denaturation at $95^{\circ} \mathrm{C}, 20 \mathrm{sec}$ of primer annealing at $58^{\circ} \mathrm{C}$ and $20 \mathrm{sec}$ of extension/synthesis at $72^{\circ} \mathrm{C}$.

In another experiment of RT-PCR for c-Src mRNA, products were electrophoresed in $1.8 \%$ agarose gel containing $0.1 \%$ ethidium bromide. Images of the fluorescent bands were captured by use of the Bio-Rad gel documentation system.

Construction and transfection of plasmid vectors. The pGPU6/green fluorescent protein (GFP)/Neo vector with a U6 promoter was used to express short hairpin RNA (shRNA) in target cells. According to the screening result, the targeting sequence is 5'-AACAAGAGCAAGCCCAAGGAT-3'. Two DNA template oligonucleotides of the shRNA were synthesized. The loop sequence of the template oligonucleotide was 5'-CAGGAGATT-3'. The hairpin cDNA were generated though annealing of the complementary oligonucleotide synthesized, where $B b s \mathrm{I}$ and $B a m \mathrm{HI}$ sites were constructed. The hairpin cDNA was subcloned into pGPU6/GFP/Neo vector at $B b s \mathrm{I}$ and BamHI sites. The recombinant pGPU6/GFP/Neo vector was called psiRNA-c-Src (Fig. 1). Subsequently it was used to transform E. coli GT116 competent cells. psiRNA-c-Src plasmids were selected and plasmid DNA was extracted and purified. The clones were verified by DNA sequencing. Plasmid psiRNA-c-Src and negative control vector psiRNA-N (empty vector pGPU6/GFP/Neo) were transfected into Panc-1 cells by Lipofectamine $2000^{\mathrm{TM}}$ respectively, according to the manufacturer's protocol (Invitrogen). Stably resistant cells were selected using G418, and then the transfected monoclone cells were chosen and expanded in cell pools for further studies. GFP was evaluated by fluorescent microscopy and flow cytometry to estimate the transfection efficiency.

Western blotting. Cells were washed twice and lysed on ice. After centrifugation, the supernatant was collected. Protein concentrations were determined by the Bio-Rad Dc protein assay system. Samples were then separated on $10 \%$ SDS-PAGE and transferred onto PVDF membrane. Membranes were blocked and incubated with primary antibodies, such as antic-Src antibody (1:100 dilution), anti $\beta$-actin antibody (1:200 dilution) at $4^{\circ} \mathrm{C}$ overnight. After 3 washes, the membranes underwent hybridization with a goat anti-mouse IgG conjugated with horseradish peroxidase (1:5,000 dilution) for $2 \mathrm{~h}$ at room temperature. After further washing, reactive bands were visualized using ECL ${ }^{\mathrm{TM}}$ western blot detection reagents with exposure to X-ray film for 30-120 sec.

MMT assayafter treatment with gemcitabine. Cells were seeded in 96-well culture plates at an optimal density $\left(5 \times 10^{3}\right.$ cells/well) in quintuplicate wells. The cells were divided into five groups. One group was not treated with the drug while the other groups were treated with chemotherapy drug, gemcitabine, in different final concentrations: $0.05,0.10,0.20$ and $0.40 \mu \mathrm{mol} / 1$. After $48 \mathrm{~h}$ of incubation, cells were stained with $20 \mu \mathrm{l} \mathrm{MTT}(5 \mathrm{mg} / \mathrm{ml})$ at $37^{\circ} \mathrm{C}$ for $4 \mathrm{~h}$ and subsequently made soluble in $150 \mu \mathrm{l}$ of DMSO. Absorbance (A) was measured at $570 \mathrm{~nm}$ using a microtitration plate spectrophotometer. Cell growth curves were calculated as mean values of each group.

Detecting VEGF levels in culture supernatants by ELISA. Cells were seeded in new cell culture bottles, and after $72 \mathrm{~h}$ culture supernatants were collected and cell counting was performed. The expression of VEGF in collected supernatants was tested by human VEGF ELISA kit (R\&D Co.) according to the handbook. VEGF concentration/cell counting was the VEGF expression level. VEGF expression level in samples of control group was considered as 1 , and the expression of VEGF in other group were calculated by comparing to the control. Duplicate wells were set, and the whole experiment was repeated twice.

Xenotransplantation of carcinoma cells. A total of $15 \mathrm{BALB} /$ c-nu mice, 5-weeks old and 20-24 g in weight were bred in an specified-pathogens free (SPF) condition and kept at a constant humidity and temperature $\left(25-28^{\circ} \mathrm{C}\right)$. The mice were divided into three groups randomly $(n=5)$. Each group underwent subcutaneous injection of $200 \mu \mathrm{l}$ cell suspension of psiRNAc-Src group, psiRNA-N group, and the control group cells in the infraaxillary region, respectively. The cell density of every 

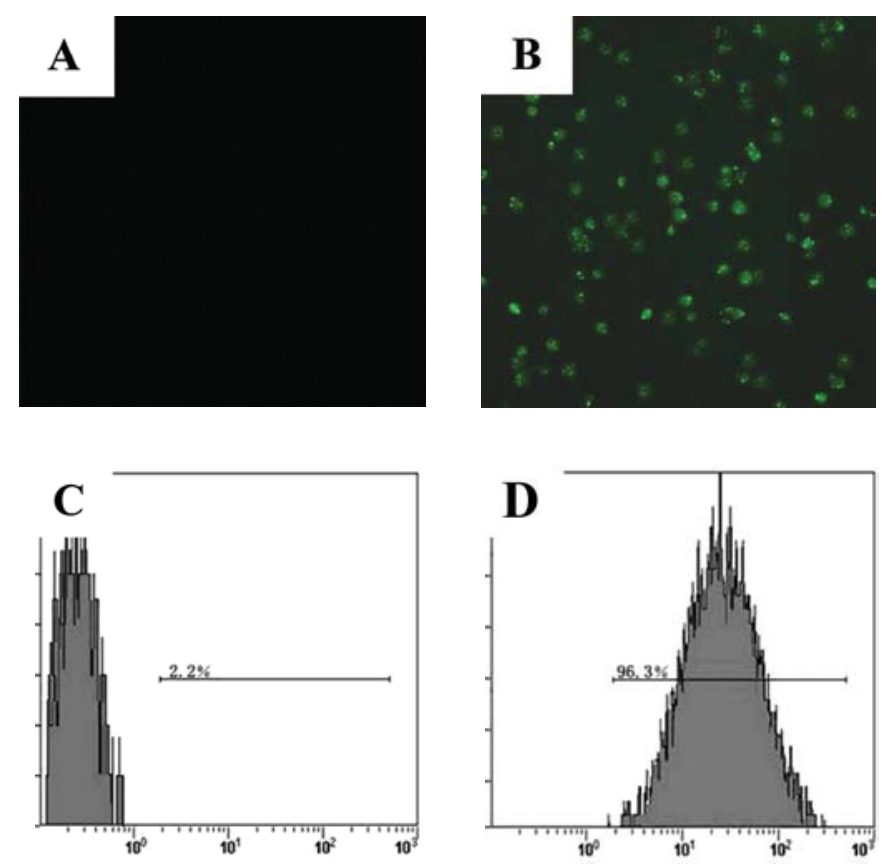

Figure 2. (A) Panc-1 cells and Panc-1 cells transfected with (B) siRNA-N labeled with FITC assessed under a fluorescent microscope. Magnification, x100. (C) Panc-1 cells and Panc-1 cells transfected with (D) siRNA-N labeled with FITC assessed by FCM. The percentage of Panc-1 cells showing fluorescence in total cells were $96.3 \%$ compared with the control $2.2 \%$. The result showed the high efficiency of siRNA transfection.

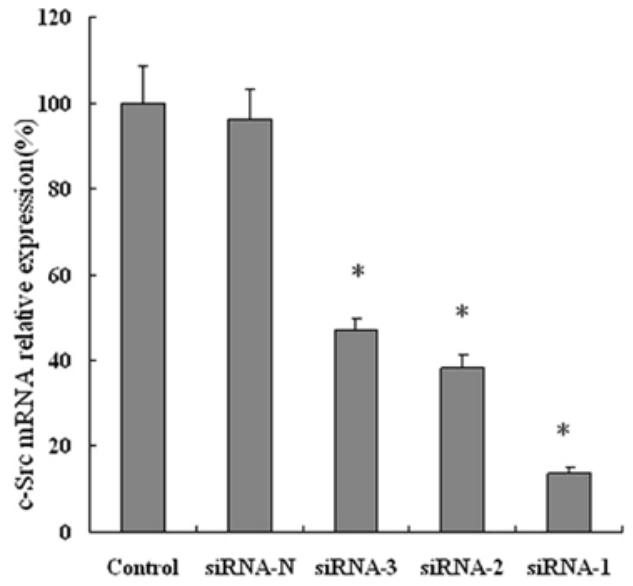

Figure 3. The knockdown effect of c-Src mRNA by real-time RT-PCR. Relative expressions of $c-S r c$ mRNA levels were analyzed using the $2^{-\Delta \Delta \mathrm{Ct}}$ method. siRNA-1, siRNA-2, siRNA-3 group "P<0.05 vs. the control group. siRNA-N group "P $>0.05$ vs. the control group. siRNA-1 were the most effective down regulating sequences, the inhibition efficiency was $86.1 \%$.

group was $2.5 \times 10^{7}$. The size of tumors was measured twice a week with calipers, and the volume was determined using the simplified formula of a rotational ellipsoid $\left(\mathrm{LxW}^{2} \mathrm{x} 0.5\right)$. Tumors were harvested from mice 5 weeks after treatment.

Immunohistochemistry. Tumor specimens were fixed in formalin overnight and embedded in paraffin. Series sections of $4 \mu \mathrm{m}$ thick were prepared for immunohistological staining. Tissue sections were quenched for endogenous peroxidase with freshly prepared $3 \% \mathrm{H}_{2} \mathrm{O}_{2}$ with $0.1 \%$ sodium azide and then placed in an antigen retrieval solution for $15 \mathrm{~min}$. After incubation in the casein block, primary antibodies such as anti-c-Src, anti-VEGF and anti-CD34 were applied to the sections for $1 \mathrm{~h}$ at room temperature, followed by incubation with the second antibody and ExtrAvidin-conjugated horseradish peroxidase. The immune reaction was counterstained with hematoxylin, dehydrated and mounted. Sections were then evaluated for the presence of brown diaminobenzidine precipitates indicative of positive reactivity by microscopy. Ten visual fields (x200 magnification) were counted for a section. The brown staining in or around the nucleus was read as positive reactivity for c-Src and VEGF. CD34 was used as the biomarker of the endothelial cell of the new blood microvessel. One lumen of blood vessels was assessed as one new blood capillary. Microvessel density was calculated by the average of microvessel count in every visual field of the section.

Statistical analysis. Each experiment was performed at least three times and found to be reproducible. Data are shown as mean \pm SD and the statistical significance of differences between the groups was determined by applying one-way analysis of variance (ANOVA), followed by Fisher's least significant difference. A P-value $<0.05$ was considered statistically significant. These analyses were performed using SPSS 13.0 software.

\section{Results}

Efficienty of siRNA transfection. Six hours after transfection with siRNA, labeled FITC Panc- 1 cells were examined by fluorescence microscope (Fig. 2A and B) and flow cytometer (FCM) (Fig. 2C and D). The percentage of Panc-1 cells showing fluorescence in total cells was $96.3 \%$ compared with the control $2.2 \%$. The result showed the high efficiency of siRNA transfection.

Screening the most effective siRNA by RT-PCR. To determine the effect of the c-Src downregulation, Real-time RT-PCR 


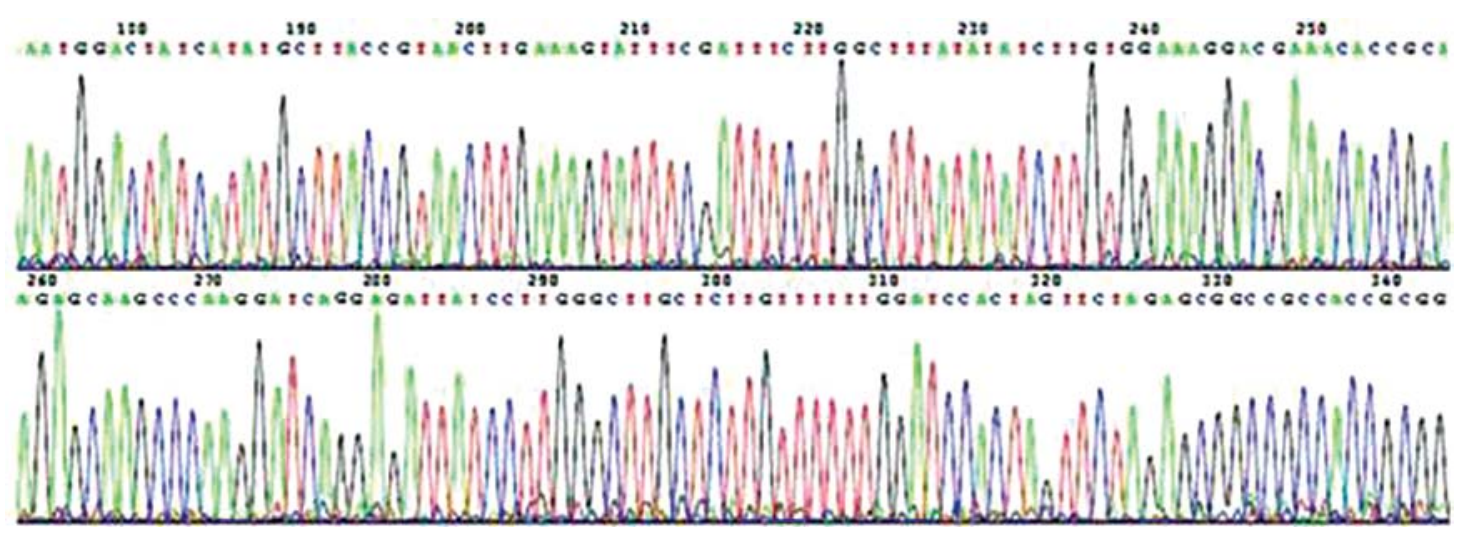

Figure 4. Parts of the sequencing result for the recombinant vector psiRNA-c-Src. The target sequences were constructed to the plasmid correctly.
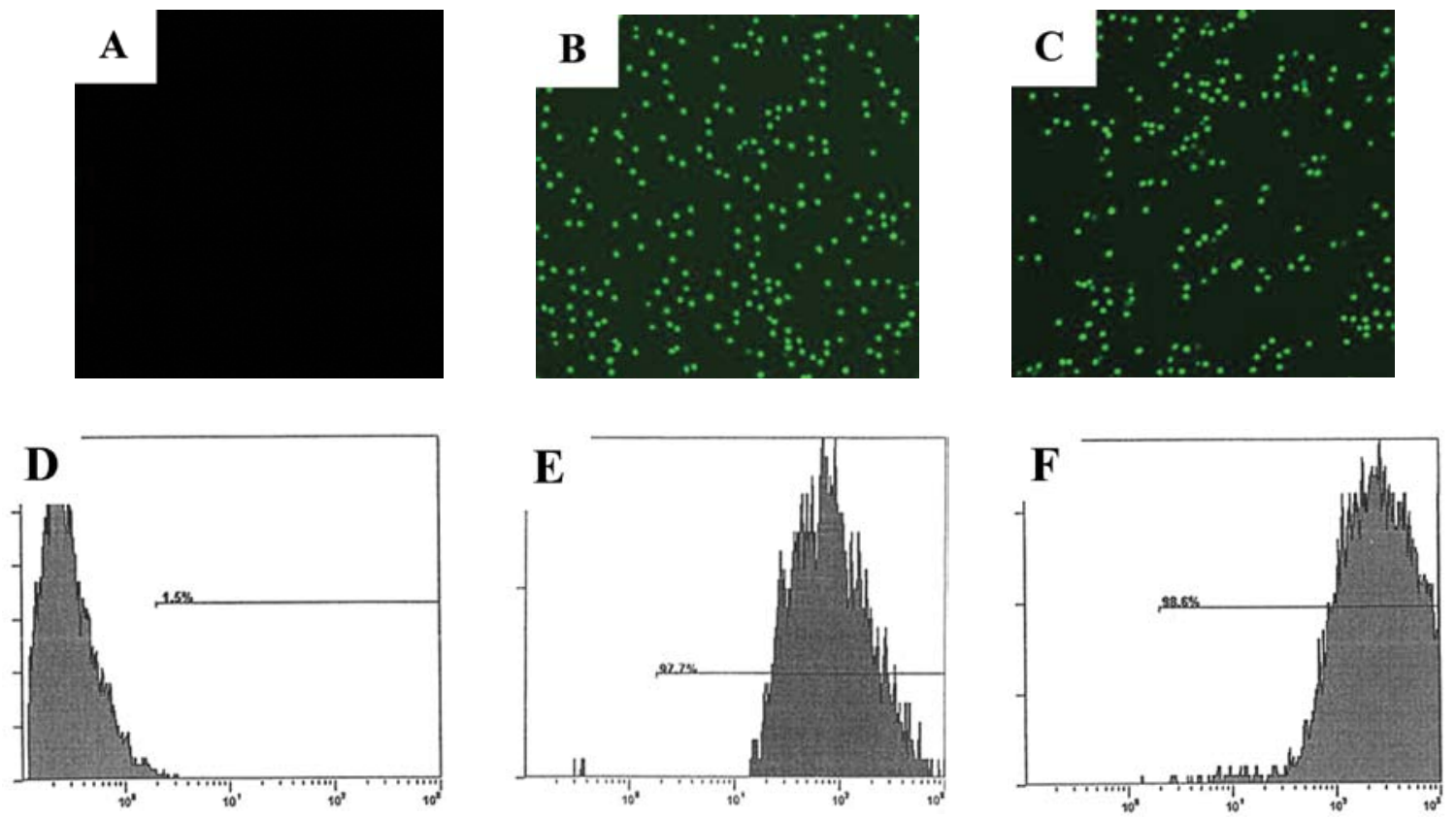

Figure 5. (A) Panc-1 cells, control group, psiRNA-N group were (B) Panc-1 cells transfected with psiRNA-N, and (C) psiRNA-c-Src group, Panc-1 cells transfected with psiRNA-c-Src GFP expression of the three groups was assessed under a fluorescent microscope. Magnification, x100. (D) Control group, (E) psiRNA-N group, (F) psiRNA-c-Src group, GFP expression was assessed by FCM. Transfection efficiency of psiRNA-N and psiRNA-c-Src were 97.7 and $98.6 \%$, respectively.

was performed to determine the mRNA levels of c-Src after transfection. After treatment with $50 \mathrm{nmol} / \mathrm{l}$ of siRNA for $48 \mathrm{~h}$, the relative c-Src mRNA levels in Panc-1 cells of different group siRNA-1, siRNA-2, siRNA-3 and siRNA-N were 13.9, $38.3,47.0 \%$ (compared with the control, ${ }^{*} \mathrm{P}<0.05$ ) and $96.2 \%$ respectively (Fig. 3). There were no significant differences between siRNA-N (negative control) group and the control group $(\mathrm{P}>0.05)$. siRNA-1 were the most effective downregulating sequences, the inhibition efficiency was $86.1 \%$.

Sequencing result and transfection efficienty of the plasmids. The result of sequencing for the recombinant vector psiRNAc-Src confirmed that the target sequences were constructed to the plasmid correctly (Fig. 4). We used a plasmid vector system to express shRNAs directed against c-Src. In addition, GFP was incorporated as a reporter gene. After transfection with the plasmids and selection with G418 for 4 weeks subsequently, we obtained G418-resistant cells. Then the transfected monoclone cells were chosen and expanded. They were examined by fluorescence microscopy (Fig. 5A-C) and FCM (Fig. 5D-F), a high percentage (>90\%) of transfectants expressed GFP, indicating a high and stable transfection of the plasmid vector system.

c-Src silencing effect assessment by RT-PCR and western blotting. To detect the silencing effect of the psiRNA-c-Src, real-time RT-PCR and western blotting were performed to determine the mRNA and protein levels of c-Src after transfection. The relative c-Src mRNA levels in the psiRNA-c-Src group and psiRNA-N group were $10.2 \%$ (compared with the control, ${ }^{*} \mathrm{P}<0.05$ ) and $100.7 \%$ (compared with the control, $\mathrm{P}>0.05$ ), respectively (Fig. 6A). In the psiRNA-c-Src group, the inhibition efficiency of c-Src mRNA was $89.8 \%$. In another experiment of RT-PCR, as shown in the electrophoretogram 

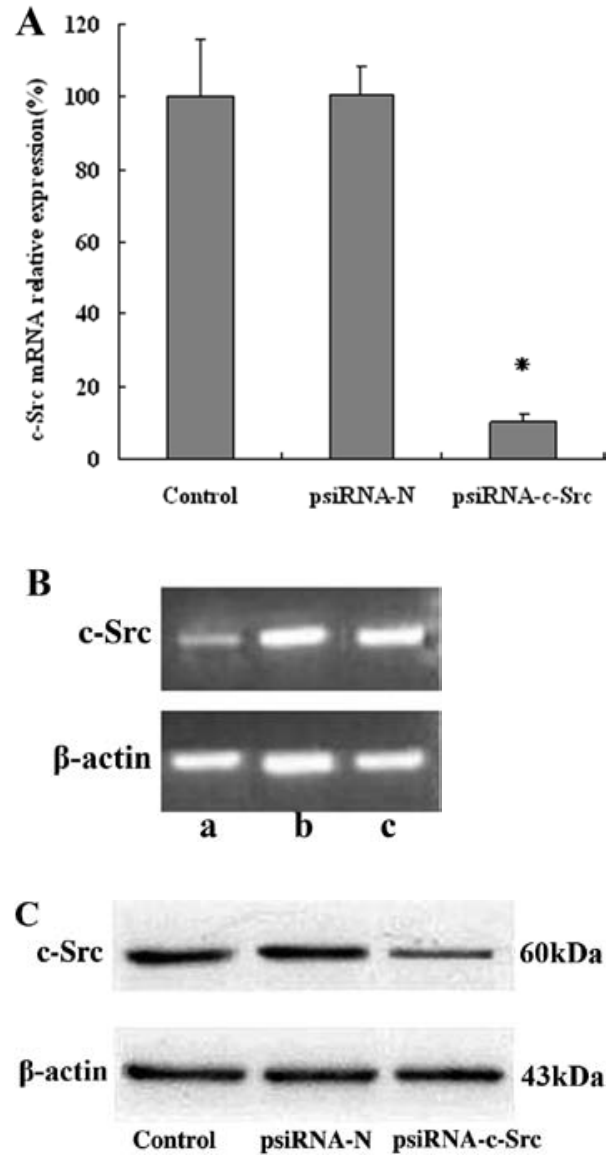

Figure 6. The silencing effect of c-Src mRNA and protein expression by (A and B) RT-PCR and (C) western blotting. (A) Relative expression of c-Src mRNA level was analyzed using the $2^{-\Delta \Delta C t}$ method. C-Src mRNA expression was significantly inhibited in the psiRNA-c-Src group ( $\mathrm{P}<0.05$ vs. the control group). (B) RT-PCR of c-Src mRNA in three groups was performed with $\beta$-actin as loading control (a, psiRNA-c-Src; b, psiRNA-N; c, control). C-Src mRNA in the psiRNA-c-Src group was knocked down vs. the other two groups. (C) Antibody specific for c-Src (molecular weight, $60 \mathrm{kDa}$ ) was used to the protein level change, while $\beta$-actin (molecular weight, $43 \mathrm{kDa}$ ) was used to act as an internal control. C-Src protein expression level was clearly downregulated vs. the other two groups.

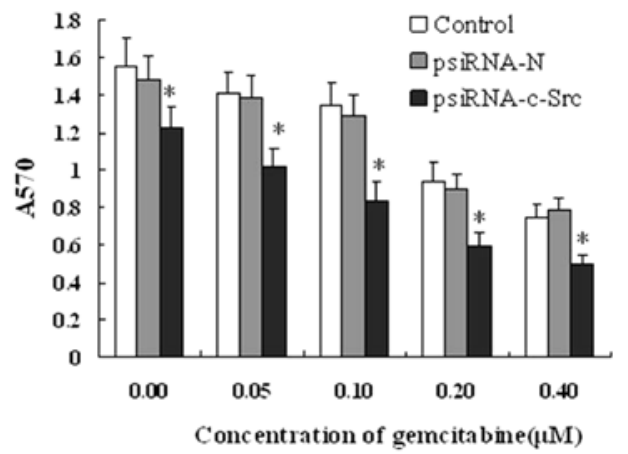

Figure 7. Cells proliferation after treatment with gemcitabine of three groups assessed by MTT assay. At each concentration of gemcitabine, the psiRNAc-Src group proliferated at a significantly lower rate than psiRNA-N group and control group $\left({ }^{*} \mathrm{P}<0.05\right)$. Inhibition of $\mathrm{c}$-Src expression enhances pancreatic carcinoma cell Panc-1 chemosensitivity to gemcitabine.

(Fig. 6B), c-Src mRNA expression in the psiRNA-c-Src group showed an obvious knockdown effect versus the other two groups. A $60 \mathrm{kDa}$ protein band, c-Src protein, was detected in

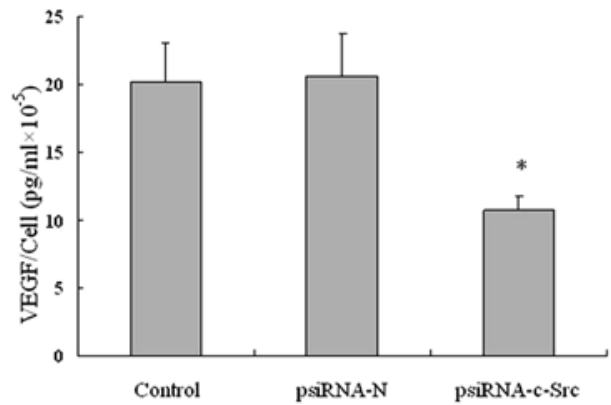

Figure 8. VEGF expression levels in vitro after inhibition of c-Src by ELISA assay. VEGF concentration/cell counting was the VEGF expression level. The VEGF expression in culture supernatants of psiRNA-c-Src group was clearly downregulated (" $\mathrm{P}<0.05)$.

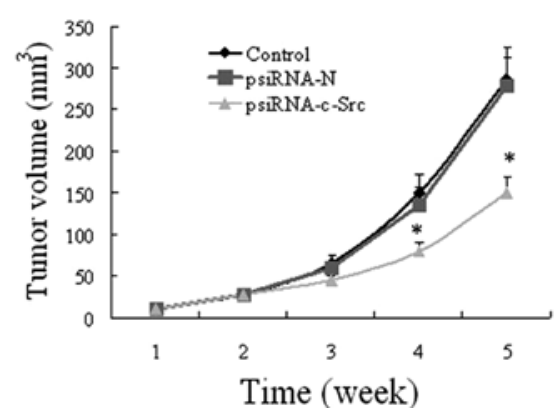

Figure 9. Inhibition of Panc-1 tumor growth in vivo. The xenotransplantation tumor volume change of the mice was detected weekly. The tumor volume of psiRNA-c-Src group was obviously lower $(" \mathrm{P}<0.05)$ than the other two groups from the fourth week.

the control group and psiRNA-N group, but weakly expressed in the psiRNA-c-Src group (Fig. 6C).

Cell proliferation by MTT assay. MTT assay was used to investigate cell proliferation in different groups after treatment with gemcitabine (Fig. 7). At each concentration of gemcitabine, the psiRNA-c-Src group proliferated at a significantly lower rate than psiRNA-N group and control group ( $\left.{ }^{*} \mathrm{P}<0.05\right)$. There was no significant difference in the growth rates between control group and the psiRNA-N group ( $\left.{ }^{*} \mathrm{P}>0.05\right)$. Inhibition of $\mathrm{c}-\mathrm{Src}$ expression enhances gemcitabine-induced cytotoxicity.

VEGF expression levels in vitro. To detect the down-regulation effect on VEGF expression after inhibition of c-Src, ELISA assay was performed. VEGF concentration/cell counting was the VEGF expression level (Fig. 8). The VEGF expression level in culture supernatants of psiRNA-c-Src group was $10.70 \pm 1.06 \mathrm{pg} / \mathrm{ml} \times 10^{-5}$, and compared with control group $\left(20.16 \pm 2.90 \mathrm{pg} / \mathrm{ml} \times 10^{-5}\right)$, it was clearly inhibited $\left({ }^{*} \mathrm{P}<0.05\right)$, with a high efficiency $(46.92 \%)$. There were no significant differences between the psiRNA-N group and the control group.

Tumor growth in vivo. All the 15 mice developed detectable tumors at the beginning of this experiment. Significant inhibition of growth was observed in psiRNA-c-Src group for 5 weeks, when compared to psiRNA-N group $\left(287 \pm 38 \mathrm{~mm}^{3}\right)$ 
A $\mathbf{a}$

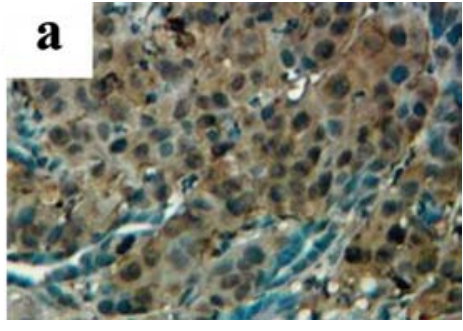

B a

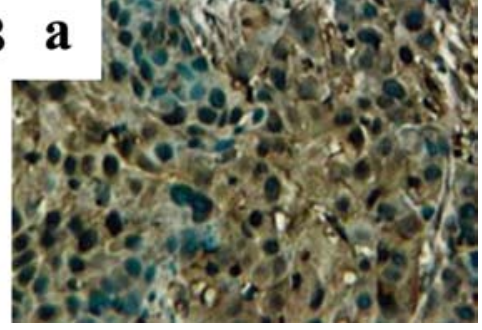

\section{C a}

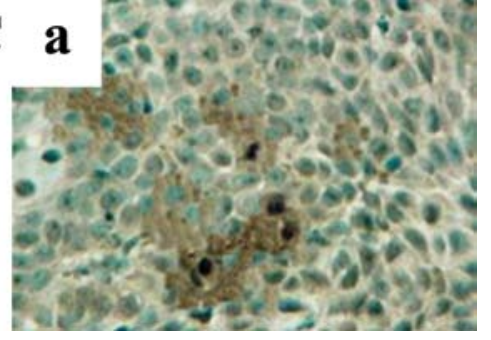

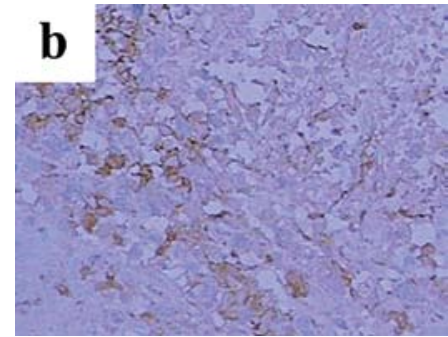
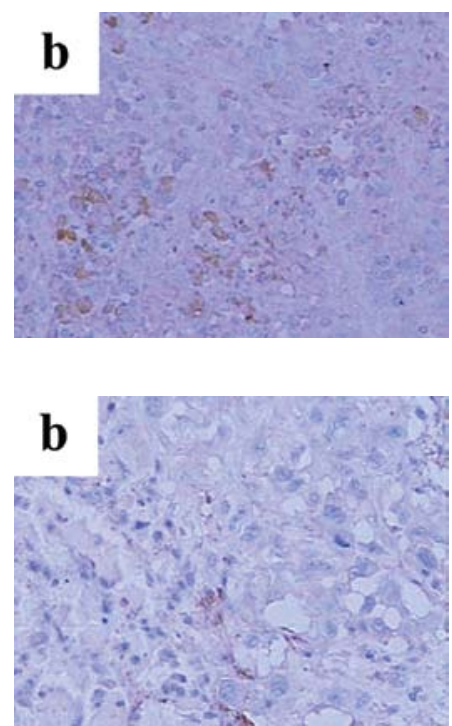
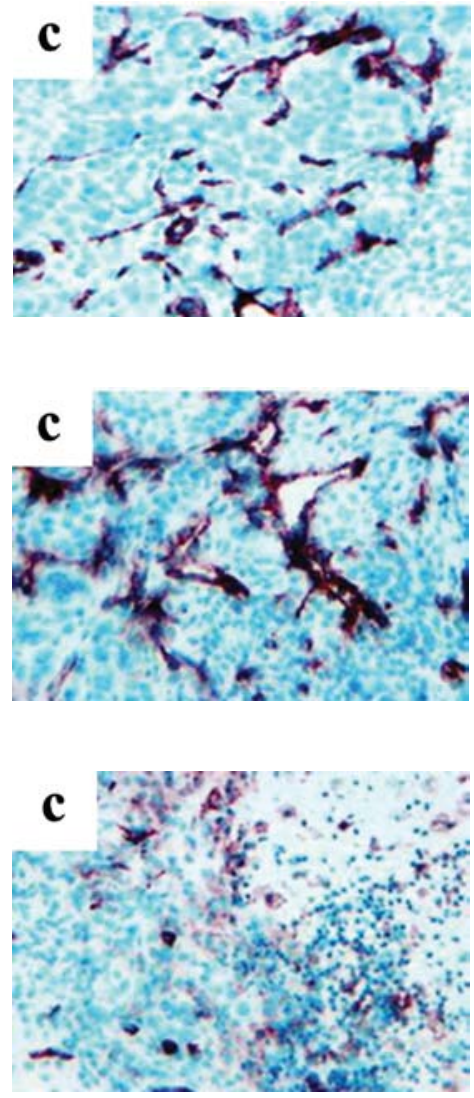

D

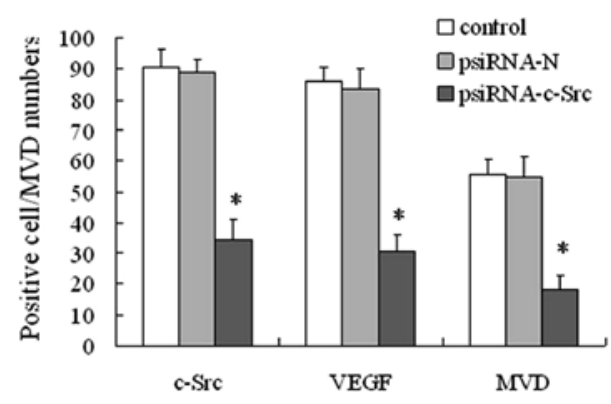

Figure 10. Expression of c-Src, VEGF and CD34 in a tumor animal model of (A) the control group, (B) psiRNA-N group, (C) psiRNA-c-Src group with different histology, as assessed by immunohistochemistry. The analysis showed (a) c-Src expression, (b) VEGF expression and (c) CD34 expression in tumor tissues. Magnification, x100. Compared with the other two groups, the expression of c-Src, VEGF and CD34 in tumor of the psiRNA-c-Src group was clearly downregulated. (D) The positive cell number of c-Src, VEGF and MVD. The expression of c-Src, VEGF and MVD in tumor of psiRNA-c-Src group was clearly downregulated ( $\mathrm{P}<0.05$ vs. the control group).

or control group $\left(278 \pm 35 \mathrm{~mm}^{3}\right)$. The average tumor volume $\left(150 \pm 20 \mathrm{~mm}^{3}\right)$ in former group was significantly lower than the latter two groups ( $\mathrm{P}<0.05)$ (Fig. 9). There were no significant differences between the psiRNA-N group and the control group.

Expression of c-Src, VEGF and CD34 by immunohistochemistry. In order to demonstrate the mechanism of the anti-angiogenic effect by targeting c-Src gene RNAi, the expression of c-Src, VEGF and CD34 were checked by immunohistochemistry in nude mice transplanted tumors. psiRNA-c-Src group (Fig. 10C) was shown to down-regulate the c-Src, VEGF and CD34 expression compared with the psiRNA-N group (Fig. 10B) and the control group (Fig. 10A) $\left({ }^{*} \mathrm{P}<0.05\right)$. There were no significant differences between the psiRNA-N group and the control group (Fig. 10) $(\mathrm{P}<0.05)$.

\section{Discussion}

Pancreatic adenocarcinoma has one of the highest mortality rates in human malignancies, accounting for more than $20 \%$ of gastrointestinal cancer deaths $(10,11)$. The lethality of pancreatic cancer is perhaps best underscored by the 5 -year survival rate, which stands at less than $4 \%$ (10). The high mortality associated with pancreatic cancer is attributed to its unusual aggressiveness. At the time of diagnosis, the disease has often progressed to an advanced stage at which surgical resection is often not a viable option and at which tumors are highly resistant to conventional chemotherapy and radiation treatments $(12,13)$. The resistance of pancreatic adenocarcinoma to conventional treatment strategies has led to a search for novel targeted therapies that may be useful in fighting this disease. 
For the low efficiency of traditional diagnosis and treatment on pancreatic cancer $(1,14)$, gene therapy has been emphasized recently for better prognosis (15). In this study, we chose plasmid pGPU6/GFP/Neo vector because it has a neomycin resistance gene, with which stable transfected cells can be selected by G418, and it can express shRNA long-term. Our results showed that high gene transduction efficiency $(>90 \%)$ was achieved after cells were selected by G418 and positive monoclones were chosen.

Recent studies have shown that c-Src, which is a $60 \mathrm{kDa}$ non-receptor tyrosine kinase product of the c-Src protooncogene and a vital member of the Src tyrosine kinase family, exhibits elevated protein levels and activity in numerous types of human cancers (16-18). Specifically, Src activity was found to be elevated in breast, pancreatic, ovarian, oesophageal, lung, gastric, colon and head and neck cancers (18-22). The frequency with which elevated expression and/or activity of Src occurs in epithelial cancers strongly suggests its implication in facilitating malignant progression. C-Src activity has been found to be a critical component of multiple signaling pathways that regulate proliferation, survival, metastasis and angiogenesis (16,23-25).

The non-receptor c-Src family of tyrosine kinases has been shown to be overexpressed and upregulated in both human pancreatic carcinoma tissue and human pancreatic tumor cell lines (26). C-Src expression and kinase activity in pancreatic cancer cell lines were directly correlated with gemcitabine chemoresistance (27). The above evidence highlighted the potential of c-Src as a target for pancreatic carcinoma. In the present study, after successful transfection by plasmid mediated c-Src RNA interference, the mRNA and protein level of c-Src gene were virtually knocked down. RNA interference is a posttranscriptional gene silencing mechanism that can be initiated by doublestranded RNA (dsRNA) homologous in sequence to the target gene $(8,9)$. Decreased expression of c-Src in human pancreatic carcinoma cell line and in xenografted tumors contributed to observe the effect of chemosensitivity to gemcitabine, angiogenesis and growth.

Our study in vitro found that inhibition of c-Src expression affected cell viability. In addition, we observe that downregulation of c-Src expression and kinase activity affects pancreatic adenocarcinoma Panc-1 cells chemosensitivity to gemcitabine and enhances gemcitabine-induced cytotoxicity, which is consistent with the report of Duxbury et al (27).

Tumor growth relies on angiogenesis, the formation of new blood vessels to receive an adequate supply of oxygen and nutrients (2,3). Angiogenesis in pancreatic carcinoma is based on the same fundamental principles of activation, proliferation, and migration of endothelial cells. Secreted angiogenic factors, such as VEGF, activate resting endothelial cells in adjacent blood vessels $(28,29)$. VEGF is important to the growth of many solid tumors conferring survival advantage by inducing vascular formation. Overexpression of angiogenic genes, such as VEGF, has been shown to be associated with enhanced tumorigenicity and tumor metastatic potential $(4,28)$. In ELISA assay in vitro and animal study in vivo, we found that plasmidbased RNAi of c-Src decreased the VEGF expression level. Additionally, CD34 is a cellsurface marker of progenitor cells and is frequently used as a new vessel marker and an indicator of microvessel density in tissues $(30,31)$. Immunostaining assays revealed CD34 in tumors was significantly decreased after psiRNA-c-Src transfection, which was concurrent with the downregulation of VEGF, gene downstream of c-Src. As shown in the experiment of xenografted tumors in mice, downregulation of angiogenesis related factors (CD34 and VEGF) may lead to the suppression of cancer growth, resulting in reduced tumor size.

In conclusion, our findings have shown that c-Src plays a significant role in pancreatic carcinoma proliferation, chemoresistance and angiogenesis. This study showed that plasmid-based c-Src specific siRNA inhibits pancreatic adenocarcinoma proliferation, angiogenesis, and enhances pancreatic adenocarcinoma gemcitabine chemosensitivity. Our findings support the theory that $\mathrm{c}$-Src is a promising target for the treatment of pancreatic carcinoma.

\section{Acknowledgements}

This study was supported by a grant from Post-Graduate Scientific Research Innovation Project of Education Department of Jiangsu Province (no. CXZZ11_0125), China, and Science and Technology Research Project of Science and Technology Bureau of Suzhou City (no. SYS201120), China.

\section{References}

1. Hidalgo M: Pancreatic cancer. N Engl J Med 362: 1605-1617, 2010.

2. Folkman J: Tumor angiogenesis: a possible control point in tumor growth. Ann Intern Med 82: 96-100, 1975.

3. Kerbel RS: Tumor angiogenesis. New Engl J Med 358: 2039-2049, 2008.

4. Dai J and Rabie A: VEGF: an essential mediator of both angiogenesis and endochondral ossification. J Dent Res 86: 937-950, 2007.

5. Midgley R, Kerr D: Bevacizumab - current status and future directions. Ann Oncol 16: 999-1004, 2005.

6. Ghaneh P, Costello E and Neoptolemos JP: Biology and management of pancreatic cancer. Postgrad Med J 84: 478-497, 2008.

7. Pan X, Sheng WH, Zhu QY, et al: Inhibition of pancreatic carcinoma growth by adenovirus-mediated human Interleukin-24 expression in animal model. Cancer Biother Radio 23: 425-434, 2008.

8. Merritt WM, Bar-Eli M and Sood AK: The dicey role of dicer: implications for RNAi therapy. Cancer Res 70: 2571-2574, 2010.

9. Agrawal N, Dasaradhi PVN, Mohmmed A, et al: RNA interference: biology, mechanism, and applications. Microbiol Mol Biol Rev 67: 657-685, 2003.

10. Korc M: Pathways for aberrant angiogenesis in pancreatic cancer. Mol Cancer 2: 1-8, 2003.

11. Shi X, Friess H, Kleeff J, et al: Pancreatic cancer: factors regulating tumor development, maintenance and metastasis. Pancreatology 1: 517-524, 2001.

12. Keleg S, Buchler P, Ludwig R, et al: Invasion and metastasis in pancreatic cancer. Mol Cancer 2: 14-20, 2003.

13. MacKenzie MJ: Molecular therapy in pancreatic adenocarcinoma. Lancet Oncol 5: 541-549, 2004.

14. Takhar AS, Palaniappan P, Dhingsa R and Lobo DN: Recent developments in diagnosis of pancreatic cancer. BMJ 329: 668-673, 2004.

15. Jimeno A and Hidalgo M: Molecular biomarkers: their increasing role in the diagnosis, characterization, and therapy guidance in pancreatic cancer. Mol Cancer Ther 5: 787-796, 2006.

16. Frame CM: Src in cancer: deregulation and consequences for cell behaviour. Biochim Biophys Acta 1602: 114-130, 2002.

17. Thomas SM and Brugge JS: Cellular functions regulated by Src family kinases. Annu Rev Cell Dev Biol 13: 513-607, 1997.

18. Summy JM and Gallick GE: Src family kinases in tumor progression and metastasis. Cancer Metastasis Rev 22: 337-358, 2003.

19. Zhang Q, Thomas SM, Xi S, et al: Src family kinases mediate epidermal growth factor receptor ligand cleavage, proliferation, and invasion of head and neck cancer cells. Cancer Res 64: 6166-6173, 2004. 
20. Golas JM, Lucas J, Etienne C, et al: SKI-606, a Src/Abl inhibitor with in vivo activity in colon tumor xenograft models. Cancer Res 65: 5358-5364, 2005.

21. Brunton VG, Avizienyte E, Fincham VJ, et al: Identification of Src-specific phosphorylation site on focal adhesion kinase: dissection of the role of Src SH2 and catalytic functions and their consequences for tumor cell behaviour. Cancer Res 65: 1335-1345, 2005.

22. Lesslie DP, Summy JM, Parikh NU, et al: Vascular endothelial growth factor receptor-1 mediates migration of human colorectal carcinoma cells by activation of Src family kinases. Br J Cancer 94: 1710-1717, 2006.

23. Schlessinger J: New roles for Src kinases in control of cell survival and angiogenesis. Cell 100: 293-296, 2000.

24. Boyer B, Bourgeois Y and Poupon MF: Src kinase contributes to the metastatic spread of carcinoma cells. Oncogene 21: 2347-2356, 2002.

25. Pories SE, Hess DT, Swenson K, et al: Overexpression of pp60csrc elicits invasive behavior in rat colon epithelial cells. Gastroenterology 114: 1287-1295, 1998.
26. Lutz MP, Esser IBS, Flossmannkast BBM, et al: Biochem Biophys Res Commun 243: 503-508, 1998.

27. Duxbury MS, Ito H, Zinner MJ, et al: siRNA directed against c-Src enhances pancreatic adenocarcinoma cell gemcitabine chemosensitivity. J Am Coll Surg 198: 953-959, 2004.

28. Girling JE and Rogers PAW: Regulation of endometrial vascular remodelling: role of the vascular endothelial growth factor family and the angiopoietin-TIE signaling system. Reproduction 138: 883-893, 2009.

29. Lewis CE, De Palma M and Naldini L: Tie2 expressing monocytes and tumor angiogenesis: regulation by hypoxia and angiopoietin-2. Cancer Res 67: 8429-8432, 2007.

30. Krause DS, Fackler MJ, Civin CI, et al: CD34: structure, biology, and clinical utility. Blood 87: 1-13, 1996.

31. Nielsen JS and McNagny KM: Novel functions of the CD34 family. J Cell Sci 121: 3683-3692, 2008. 